\title{
Central diabetes insipidus and pituitary stalk thickening in adults: distinction of neoplastic from non-neoplastic lesions
}

\author{
France Devuyst', Paraskevi Kazakou², Danielle Balériaux ${ }^{3}$, Orsalia Alexopoulou ${ }^{4}$, Agnès Burniat', \\ Sylvie Salenave5, Philippe Chanson ${ }^{5,6}$, Bernard Corvilain' ${ }^{1}$ and Dominique Maiter ${ }^{4}$
}

'Department of Endocrinology, Erasme Hospital, Université libre de Bruxelles, Brussels, Belgium, ${ }^{2}$ Endocrine Unit, Department of Medical Therapeutics, Alexandra Hospital, National and Kapodistrian University of Athens, Athens, Greece, ${ }^{3}$ Department of Neuroradiology, Erasme Hospital, Université libre de Bruxelles, Brussels, Belgium, ${ }^{4}$ Department of Endocrinology and Nutrition, Université Catholique de Louvain, Cliniques Universitaires Saint-Luc, Brussels, Belgium, ${ }^{5}$ Assistance Publique-Hôpitaux de Paris, Hôpital Bicêtre, Service d'Endocrinologie et des Maladies de la Reproduction, Centre de

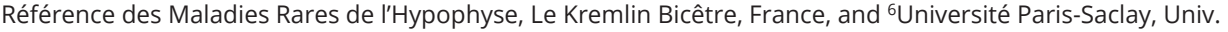
Paris-Sud, Inserm, Signalisation Hormonale, Physiopathologie Endocrinienne et Métabolique, Le KremlinBicêtre, France

Correspondence should be addressed to F Devuyst Email

fdevuyst@ulb.ac.be

\begin{abstract}
Context: Association of central diabetes insipidus (CDI) and pituitary stalk thickening (PST) may have several etiologies (including malignancies) and differential diagnosis remains often difficult.

Objective: The purpose of this study was to identify which clinical, biochemical or radiological features could help clinicians to make an etiological diagnosis, especially distinguishing neoplastic from non-neoplastic pituitary stalk lesions.

Design and methods: We retrospectively analyzed clinical, biochemical, radiological and histological data of 38 adult patients diagnosed with CDI and PST of proven etiology.

Results: Of the 38 pituitary stalk lesions included, 11 (29\%) were neoplastic. A histopathological diagnosis was obtained in 22/38 (58\%) patients. The three most frequently observed etiologies of PST were neuroinfundibulitis (34\%), germinoma (21\%) and histiocytosis (18\%). Pituitary stalk thickness was larger for neoplastic lesions, particularly germinomas. Male gender and a very young age were statistically associated with a risk of germinoma. At least one anterior pituitary deficit was observed in nearly $60 \%$ of patients. Patients with neoplastic PST were more affected by multiple anterior pituitary dysfunction than patients with benign PST. A high serum prolactin level was individually the best predictor of a neoplastic origin ( $90 \%$ sensitivity and $60 \%$ specificity for a serum prolactin level 1.27 -fold above the normal upper limit (ULN)).

Conclusion: We confirm a relatively high risk of malignancy in adult patients presenting with the association of CDI and PST. Young age, male gender, a very large thickening of the stalk, multiple anterior pituitary deficits and prolactin above $1.3 \times$ ULN increase the likelihood of a neoplastic origin.
\end{abstract}

\section{Introduction}

Adult patients with pituitary stalk lesions commonly present with varying degrees of central diabetes insipidus (CDI), hypopituitarism, and hyperprolactinemia. In particular, the association of CDI and pituitary stalk thickening (PST) may be caused by several diseases, including neoplastic, infectious, and inflammatory
Published by Bioscientifica Ltd. 
conditions $(1,2,3,4,5,6,7,8,9,10,11)$, and differential diagnosis is mandatory in order to propose an appropriate treatment and follow-up. This diagnosis remains challenging, principally for two reasons: (i) there are few specific signs on MRI, which may help to identify the underlying disease (3) and (ii) biopsies of lesions in this area are not often performed as they may be associated with significant morbidity such as cerebrospinal fluid (CSF) leak, meningitis or permanent panhypopituitarism (12). As a result, the diagnosis of the underlying disease is frequently based only on indirect clues driven by clinical and biochemical evaluation, CSF analysis, imaging, biopsy of other involved organs and/or the natural course of the disease. Moreover, few studies have specifically addressed the issue of PST associated with diabetes insipidus in children $(4,5,6,7,13)$ and even fewer in predominantly adult populations (14).

In the present study we retrospectively analyzed clinical, biochemical, radiological and histological data of 38 adult patients first diagnosed with CDI and PST and in whom evaluation led to a well-identified etiology. Given the risks associated with a pituitary stalk biopsy, we tried to highlight which clinical, biochemical or radiological data could help clinicians to make an early and accurate diagnosis, with a special emphasis on the differentiation between neoplastic and non-neoplastic lesions.

\section{Subjects and methods}

We retrospectively included all known adult patients with the association of central diabetes insipidus and pituitary stalk thickening at diagnosis. These patients had been followed between 1991 and 2016 in one of three academic centers in Belgium and France (Brussels SaintLuc University Hospital, ULB Erasme Hospital and Bicêtre University Hospital). Most patients were diagnosed after 2009 , as we started a pituitary case registration in a more systematic way since that year (2-5 patients included annually between 2009 and 2016).

Several patients were excluded from our analysis: those with a known pre-existing diagnosis at the time of CDI onset (including many patients with metastases from a previous primary cancer and patients with another known pituitary lesion such as Rathke's cleft cyst, craniopharyngioma or apoplexy), patients younger than 15-year-old at diagnosis, patients with pituitary radiation therapy or surgery without a prior available diagnostic MRI, patients without a full MRI evaluation including sagittal and coronal, nonenhanced and contrast-enhanced T1-weighted images of the hypothalamo-hypophyseal tract, and patients with insufficient diagnostic or follow-up data. Thirty-eight patients (20 males and 18 females) were eligible and their clinical, biochemical and radiological data were reviewed. The median follow-up period was 55 months (range 6-238). We also recorded histological findings when available, including results from biopsies of the pituitary stalk or of a cranial or extracranial site involved by the disease. All different investigations performed in the patients were also reviewed, in particular those allowing the exclusion of germ cell tumor or other malignancy and/or those confirming the suspected underlying disease. The most frequently performed investigations were chest X-rays or thoracic CT (34/38), serum tumor markers for germ cell tumor ( $\alpha$-fetoprotein, $\beta$ - human chorionic gonadotropin (HCG); 24/38), CSF analysis (20/38), bone scintigraphy (17/38), abdominal CT (17/38) and fluoro-deoxy-glucose (FDG)-PET coupled with CT scan (13/28).

\section{Pituitary MRI evaluation}

All MRI scans were retrospectively analyzed by the same neuroradiologist, who was blinded against the final etiological diagnosis. The pituitary stalk lesions were characterized by reviewing the first MRI that identified a pituitary stalk abnormality. Quantitative measurements of the anatomical structures were made using the scales provided on the images. The following radiological features were recorded: the presence or absence of the posterior hypophyseal bright spot; the pituitary size (the height of the anterior pituitary was measured on sagittal T1-weighted images) and the gland was characterized as normal or enlarged (height $>7 \mathrm{~mm}$ ); the width of the pituitary stalk, measured in its proximal, medial and distal portions on both coronal and sagittal sequences; the morphology of the stalk (characterized as tubular, V-shaped, round or pyramidal according to the classification reported by Turcu et al. (3)); and the enhancement pattern of the pituitary stalk (homogenous/ non-homogenous). Based on the previously reported normal dimensions of the hypothalamic-pituitary axis on MRI $(15,16,17,18)$, the pituitary stalk was considered enlarged when its largest diameter was greater than 4.0 $\mathrm{mm}$ in its proximal portion at the level of the optic chiasm, $2.8 \mathrm{~mm}$ in its medial portion and/or $2.5 \mathrm{~mm}$ in its distal portion at the level of pituitary insertion. A similar evaluation was made on subsequent MRI scans to detect any changes regarding the width of pituitary stalk. Changes were considered significant when a $\geq 25 \%$ variation in stalk diameter was observed. 


\section{Endocrine evaluation}

The following data were collected from medical records at diagnosis and during follow-up: baseline serum IGF1, LH, FSH, testosterone or estradiol, prolactin, $\mathrm{TSH}$, free thyroxine (fT4), morning cortisol and plasma adrenocorticotropin (ACTH), as well as results of dynamic function tests when available.

Secondary hormonal deficiencies were defined based on low levels of the primary hormones with low or inappropriately normal corresponding pituitary hormones. In some cases, stimulation tests were performed to confirm corticotropic or somatotropic insufficiency. CDI was diagnosed based on clinical signs and symptoms and the measurement of serum and urine osmolality and electrolytes. Some patients underwent a water deprivation test. Noticeably, the posterior pituitary bright spot was absent on T1-weighted MRI in all patients and all responded successfully to treatment with desmopressin.

\section{Statistical analysis}

The statistical analyses were performed using IBM SPSS Statistics, version 24.0 software. The continuous variables were reported as mean $\pm \mathrm{SD}$, or as median and (P5-P95) interval, depending on the normality of the data which was tested by the Shapiro-Wilk test. Continuous normally distributed variables were compared using Student's unpaired $t$-tests, whereas discrete variables were compared by the chi-square test. Correlations were calculated using Spearman regression analysis and ROC curve analysis was performed to determine the best values differentiating malignant from benign etiologies of CDI and PST. A value of $P<0.05$ was considered statistically significant.

\section{Ethical committee}

This study was approved by the Ethics Committee of Erasme Hospital.

\section{Results}

\section{General characteristics of the patients}

Thirty-eight patients (20 men and 18 women; sex ratio 1.1) were included in this study. Table 1 shows the several etiologies of association of CDI and PST. A histopathological diagnosis was obtained in 22/38 (58\%) patients. This was based on the biopsy of the pituitary stalk $(n=7)$, of an associated brain lesion $(n=4)$ or of a lesion present in extracranial tissues $(n=11)$. In the remaining 16 patients, diagnosis relied on CSF analysis in one patient (germinoma), on clinical and biological grounds in three cases (granulomatosis with polyangiitis, acute myeloid leukemia, idiopathic granuloma) and on the clinical context and spontaneously favorable evolution in 12 cases of neuroinfundibulitis.

Among the benign etiologies $(n=27), 13$ patients had neuroinfundibulitis (including two cases with panhypophysitis), seven had histiocytosis (classified as benign according to the Histiocyte Society classification (19) as all were of the ' $\mathrm{L}$ ' type and none of the ' $\mathrm{M}$ ' type), three suffered from Wegener polyangiitis (3/27), two from idiopathic granuloma, one from sarcoidosis and one

Table 1 Etiology of diabetes insipidus and pituitary stalk (PS) thickening in the 38 patients and corresponding features leading to diagnosis.

\begin{tabular}{l}
\hline Etiology \\
\hline Neuroinfundibulitis \\
Histiocytosis** \\
Germinoma \\
Wegener \\
Idiopathic granuloma \\
Burkitt lymphoma \\
Breast cancer metastasis \\
Leukemia \\
Sarcoidosis \\
Epidermoid cyst
\end{tabular}

\begin{tabular}{c}
\hline $\begin{array}{c}\text { No. of } \\
\text { patients }\end{array}$ \\
\hline 13 \\
7 \\
8 \\
3 \\
2 \\
1 \\
1 \\
1 \\
1 \\
1 \\
\hline
\end{tabular}
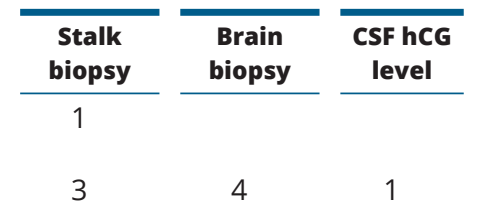

1

1

1

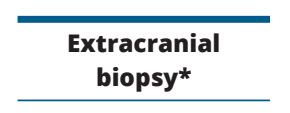

7

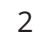

1

1

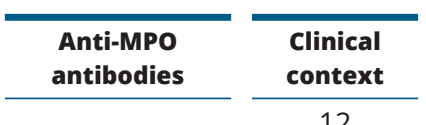

12

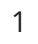

1

1

*Extra cranial biopsies were performed at the level of the skin $(n=5)$, bone $(n=2)$ for histiocytosis), kidney $(n=1)$ and sinus $(n=1)$ for Wegener's disease, adrenal $(n=1)$ for Burkitt lymphoma and salivary gland $(n=1)$ for sarcoidosis. **Patients with histiocytosis $(n=7)$ were all classified as having a benign disease according to the Histiocyte Society classification (19), as all were of the ' $L$ '-type and none of the ' $\mathrm{M}$ ' type.

anti MPO, anti-myeloperoxydase antibodies; CSF, cerebrospinal fluid; hCG, human chorionic gonadotrophin; No., number. 
from a stalk epidermoid cyst. The neoplastic etiologies were germinoma (8/11), metastatic breast cancer (1), leukemia (1) and Burkitt lymphoma (1). As shown, the three most frequently observed etiologies of PST in our series were neuroinfundibulitis (34\%) germinoma (21\%) and histiocytosis (18\%).

CDI was diagnosed at a median age of 31 years (range 15-86 years). The diagnosis of CDI was not synchronous with that of the underlying disease in nine patients (24\%). It preceded the etiological diagnosis by a delay ranging from 1 to 3 years in seven cases (four germinomas, three histiocytosis), while in two cases CDI occurred 2 years after the diagnosis (one patient with sarcoidosis and one with leukemia).

Some clinical data were useful for the etiological diagnosis. Cutaneous lesions were almost always present in patients with histiocytosis (6/7 patients) but never for other diseases. Regarding MR images, no specific feature of the pituitary stalk appearance was helpful to diagnose the underlying disease, but three out of the eight patients with a germinoma had a concurrent typical pineal lesion.

A lumbar puncture was performed in 20 patients and contributed to the diagnosis of germinoma in $6 / 8$ patients harboring this condition: AFP and hCG were measured in 15 CSF samples: AFP was negative in all, hCG was elevated in the cerebrospinal fluid (CSF) in four patients and neoplastic germinomatous cells were present in two others. Serum tumors markers (AFP and hCG) were analyzed respectively in 23 and 24 patients: AFP was negative in all. hCG was elevated in peripheral blood in three patients. Two patients had both positive serum and CSF hCG at diagnosis.

A ${ }^{18}$ F-FDG-PET-CT scan was performed in the diagnosis evaluation of the more recent cases between 2009 and 2016. This imaging modality was indeed a useful tool to confirm diagnosis, assess disease activity and staging in histiocytosis detecting hypermetabolic extracerebral manifestations of the disease, mostly bone lesions in our patients. ${ }^{18}$ F-FDG PET-CT also played a key role in the diagnosis work up of two difficult cases, guiding renal and adrenal biopsies in unusual presentations of Wegener and Burkitt lymphoma ('bifocal' localization of PST and bilateral renal pseudotumor in the first case, PST and bilateral adrenal involvement without peripheral lymph nodes in the second one).

In all patients, central diabetes insipidus was the primary clinical manifestation leading to first medical check-up.

At least one deficit of anterior pituitary hormones was observed in 22 patients (58\%), whereas hyperprolactinemia was observed in 24 (77\%) of the 31 cases in whom PRL was measured, with a prolactin range of 0.35 to 6.25 times the upper limit of normal $(\times \mathrm{ULN})$.

\section{Characteristics of the patients according to the malignant nature of underlying disease}

\section{Clinical and radiological findings}

When patients were subdivided into those with a benign etiology $(27 / 38 ; 71 \%)$ and those with a malignant disease $(11 / 38 ; 29 \%)$, mean age at diagnosis of DI was not different between the two groups $(38.7 \pm 17.2$ vs $32.7 \pm 22.4$ years $)$ but the sex ratio $(\mathrm{M} / \mathrm{F})$ was much higher at 4.5 for malignant disease (7/1 for germinoma) than for benign disease $(0.7 ; P=0.02)$ (Table 2$)$. Interestingly enough, the mean age at diagnosis of DI was lower for germinoma $(22.6 \pm 3.8$ years) than for histiocytosis $(35.0 \pm 14.5$ years) and for neuroinfundibulitis $(39.1 \pm 13.8$ years; $P<0.05)$.

Regarding clinical signs and symptoms, headaches, visual defects, other neurological symptoms and systemic manifestations were more frequent in the malignant subgroup, but the difference was only slightly significant for neurological signs (memory loss and dizziness), which were present only in the germinoma subgroup. As mentioned, cutaneous lesions were only present in patients with histiocytosis.

Pituitary stalk thickness was larger in patients with a neoplastic etiology of PST, and this difference reached statistical significance in sagittal view (median: $5.3 \mathrm{~mm}$

Table 2 General characteristics of the patients at diagnosis according to the benign or malignant nature of underlying disease.

\begin{tabular}{|c|c|c|c|}
\hline & $\begin{array}{c}\text { Benign } \\
\text { disease }(n=27)\end{array}$ & $\begin{array}{c}\text { Malignant } \\
\text { disease }(n=11)\end{array}$ & $P$ value \\
\hline $\begin{array}{l}\text { Age at diagnosis of DI } \\
\text { (years) }\end{array}$ & $38.7 \pm 17.2$ & $32.7 \pm 22.4$ & 0.378 \\
\hline Sex ratio $(\mathrm{M} / \mathrm{F})$ & $11 / 16$ & $9 / 2$ & 0.021 \\
\hline $\begin{array}{l}\text { Duration of follow-up } \\
\text { (months) }\end{array}$ & $48(2-240)$ & $60(3-204)$ & 0.890 \\
\hline \multicolumn{4}{|l|}{ Main symptoms } \\
\hline Headaches (\%) & $8 / 26(31 \%)$ & $6 / 10(60 \%)$ & 0.107 \\
\hline Visual disturbances (\%) & $5 / 26(19 \%)$ & $5 / 11(45 \%)$ & 0.101 \\
\hline $\begin{array}{l}\text { Systemic } \\
\text { manifestations (\%) }\end{array}$ & $10 / 27(37 \%)$ & $7 / 10(70 \%)$ & 0.074 \\
\hline $\begin{array}{l}\text { Cutaneous } \\
\text { manifestations (\%) }\end{array}$ & 6/26 (23\%) & $0 / 11(0 \%)$ & 0.082 \\
\hline $\begin{array}{l}\text { Other neurologic } \\
\text { symptoms (\%) }\end{array}$ & $1 / 24(4 \%)$ & $3 / 10(30 \%)$ & 0.030 \\
\hline $\begin{array}{l}\text { Stalk biopsy } \\
\text { performed (\%) }\end{array}$ & $3 / 27(11 \%)$ & $4 / 11(36 \%)$ & 0.069 \\
\hline $\begin{array}{l}\text { Lumbar puncture } \\
\text { performed (\%) }\end{array}$ & $11 / 27(41 \%)$ & $9 / 11(82 \%)$ & 0.021 \\
\hline
\end{tabular}


(range: 3.5-11.0) in neoplastic PST vs $4.0 \mathrm{~mm}$ (2.0-9.1) in benign PST; $P=0.016$; Table 3$)$. This parameter was also much larger for germinoma (5.50 mm (3.50-11.00)) than for neuroinfundibulitis $(4.30 \mathrm{~mm}(2.00-6.80))$ and histiocytosis $(3.20 \mathrm{~mm}(2.63-4.80))(P<0.01)$.

\section{Endocrine evaluation}

Partial or complete anterior hypopituitarism was more frequently observed in patients with malignant (11/11) than in non-malignant PST $(11 / 27 ; P=0.001)$. In patients with malignant PST, somatotropic and gonadotropic insufficiencies were the most common endocrine deficiencies in evaluable patients (8/9 and 9/10, respectively), followed by central hypothyroidism $(8 / 11)$ and corticotropic insufficiency (7/11) (Table 4). In patients with non-malignant PST, secondary hypogonadism was the most common endocrine deficiency in tested patients (10/25) followed by growth hormone (4/13), TSH $(5 / 26)$ and ACTH deficiencies $(2 / 23) \quad(P<0.01$ vs malignant disease for each hormonal axis). Overall, patients with neoplastic PST were more affected by multiple anterior pituitary dysfunction than patients with benign PST (Fig. 1).

The median PRL level $(\times \mathrm{ULN})$ was much higher for patients with neoplastic PST (3.10 $\times$ ULN (0.75-6.25) than for patients with benign PST $(1.15 \times$ ULN (0.353.02) (Fig. $2 ; P=0.001)$. It was also higher for germinoma $(2.79 \times \mathrm{ULN}(0.75-4.62))$ than for histiocytosis $(1.15$ $\times$ ULN\% (0.70-1.78)) and neuroinfundibulitis $(1.00 \times \mathrm{ULN}$ $(0.35-2.81))(P<0.01)$. A non-significant correlation was observed between the thickness of pituitary stalk and the level of PRL $(r=0.363, P=0.063)$. A ROC curve analysis also showed that at a cut off value for serum prolactin of $1.27 \times$ ULN could predict malignancy with a sensitivity of $90 \%$ and a specificity of $67 \%$, while a value of $2.00 \times \mathrm{ULN}$ predicted a malignant disease with a sensitivity of $70 \%$ but a high specificity of 95\% (Fig. 3; $\mathrm{AUC}=0.852 ; P=0.002$ ). Moreover, the etiology was always benign when PRL was $\leq 0.74 \times \mathrm{ULN}$ and always malignant for prolactin $\geq 3.1$ $\times \mathrm{ULN}$.

\section{Stalk biopsy}

To confirm the diagnosis, a stalk biopsy had to be performed in 3/27 patients with a benign disease (one case each of neuroinfundibulitis, idiopathic granuloma and epidermoid cyst) and in four of the 11 patients with a neoplastic disease (germinoma in three and metastasis in one patient) (Table 1). In four other cases of germ cell tumor, the biopsy could be performed stereotactically in a tumoral extension within the hypothalamus.

\section{Follow-up}

After diagnosis, a follow-up adapted to the underlying diagnosis was proposed to all patients and an appropriate treatment was given if available. Twenty-four patients were treated for the underlying disease: 14/27 (52\%) patients with benign PST and 10/11 (91\%) patients with neoplastic PST. A follow-up MRI was available for 33/38 patients. At the end of the observation period, we observed a pituitary stalk normalization in 23/33 patients (70\%): 14/24 (58\%) with a benign etiology and 9/9 (100\%) with a malignant etiology, respectively. Normalization of PST was observed after 31 (5-206) months for benign PST and 7 months (3-18) for malignant PST $(P=0.02)$.

In the neuroinfundibulitis group, follow-up MRI was available for $11 / 13$ patients and four of them had been treated with corticosteroids (Table 5). The efficacy of this treatment on PST was not clearly apparent. In the four patients treated with steroids, we observed a normalization of PST in two and a reduction in the other two patients. One of them showed a biphasic evolution with normalization of PST under corticosteroids trial followed by secondary enlargement after withdrawal of treatment. On the other hand, we observed a normalization of pituitary stalk in all seven untreated patients. The patients with a diagnosis

Table 3 Main MRI characteristics of the patients at diagnosis according to the malignant nature of underlying disease.

\begin{tabular}{|c|c|c|c|}
\hline & Benign disease $(n=27)$ & Malignant disease $(n=11)$ & P value \\
\hline Stalk thickness (coronal view) (mm) * & $4.1(2.4-10.8)$ & $5.5(3.0-22.4)$ & 0.068 \\
\hline Stalk thickness (sagittal view) (mm) * & $4.0(2.1-9.1)$ & $5.3(3.5-11.0)$ & 0.016 \\
\hline Pituitary stalk aspect (T/R/V/P) & $9 / 14 / 3 / 1$ & $5 / 4 / 2 / 0$ & 0.703 \\
\hline Stalk contrast enhancement (homo./hetero) (h§ $^{\S}$ & $17 / 5$ & $8 / 2$ & 0.863 \\
\hline Pituitary gland aspect (N/E) $)^{\S \S \S}$ & $19 / 6$ & $6 / 4$ & 0.292 \\
\hline
\end{tabular}

*Stalk thickness was recorded at the level of the largest size of the stalk on both coronal and sagittal views. ${ }^{\S}$ Pituitary stalk aspect: $\mathrm{T}$, tubular; R, round; $\mathrm{V}$, V-shape; P, pyramidal. ${ }^{\S \S}$ Contrast enhancement (data available for 32 patients): homo., homogeneous; hetero, heterogeneous. ${ }^{\S \S \S}$ Pituitary gland aspect (data available for 35 patients): N, normal; E, enlarged. 
Table 4 Endocrine evaluation of the patients at diagnosis according to the benign or malignant nature of underlying disease.

\begin{tabular}{|c|c|c|c|}
\hline & $\begin{array}{l}\text { Benign disease } \\
\quad(n=27)\end{array}$ & $\begin{array}{c}\text { Malignant } \\
\text { disease }(n=11)\end{array}$ & $P$ value \\
\hline $\begin{array}{l}\mathrm{Nb} \text { with LH/FSH } \\
\text { deficiency (\%) }\end{array}$ & $10 / 25(40 \%)$ & 9/10 (90\%) & 0.007 \\
\hline $\begin{array}{l}\mathrm{Nb} \text { with } \mathrm{GH} \\
\quad \text { deficiency (\%) }\end{array}$ & 4/13 (31\%) & $8 / 9(89 \%)$ & 0.007 \\
\hline $\begin{array}{l}\mathrm{Nb} \text { with TSH } \\
\quad \text { deficiency (\%) }\end{array}$ & $5 / 26(19 \%)$ & $8 / 11(73 \%)$ & 0.002 \\
\hline $\begin{array}{l}\mathrm{Nb} \text { with } \mathrm{ACTH} \\
\text { deficiency (\%) }\end{array}$ & $2 / 23(9 \%)$ & 7/11 (64\%) & 0.001 \\
\hline Prolactin (xULN) & $1.15(0.35-3.02)$ & $3.10(0.75-6.25)$ & $<0.001$ \\
\hline $\begin{array}{l}\text { No. of AP } \\
\text { deficits } \\
(0 / 1 / 2 / 3 / 4)\end{array}$ & $16 / 5 / 2 / 2 / 2$ & $0 / 1 / 3 / 2 / 5$ & 0.003 \\
\hline
\end{tabular}

AP, anterior pituitary; No., number; ULN, upper limit of normal.

of germinoma were treated by conventional combination of chemo- and radiotherapy, and we observed a normalization of the pituitary stalk in all of them.

In the histiocytosis group, follow-up MRI was available for $7 / 7$ patients and five of them were treated. For the five treated patients, we observed a normalization of PST in two patients and a reduction of thickening in three others. For the untreated patients: we observed a

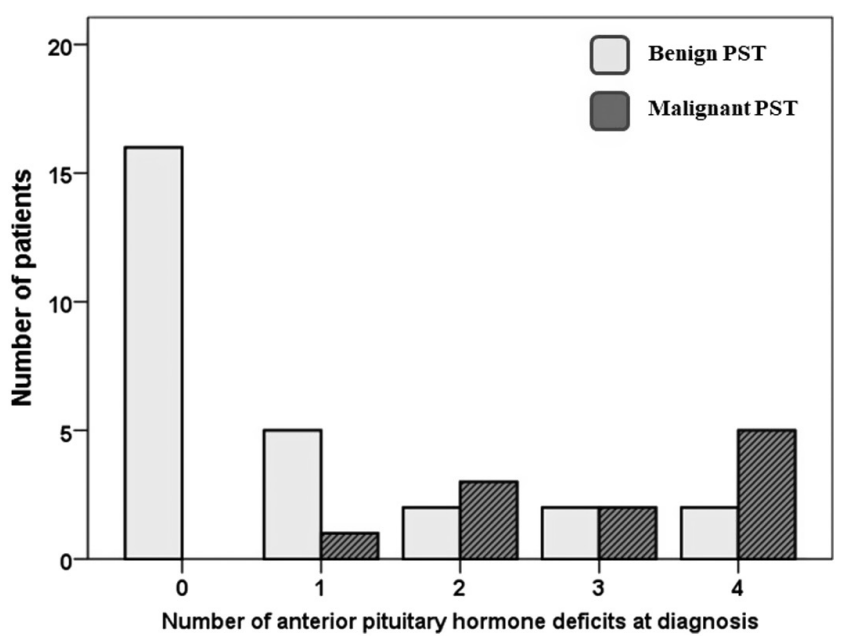

Figure 1

Numbers of patients with 0, 1, 2, 3 or 4 pituitary hormone deficits in addition to central diabetes insipidus at diagnosis. Patients with a benign etiology of pituitary stalk thickening (PST) are indicated in open bars and patients with a malignant etiology are indicated in dark grey bars. The absence of any deficit was only observed in patients with benign PST, while patient with a neoplastic PST were more often affected by multiple anterior pituitary deficiencies $(P<0.01)$.

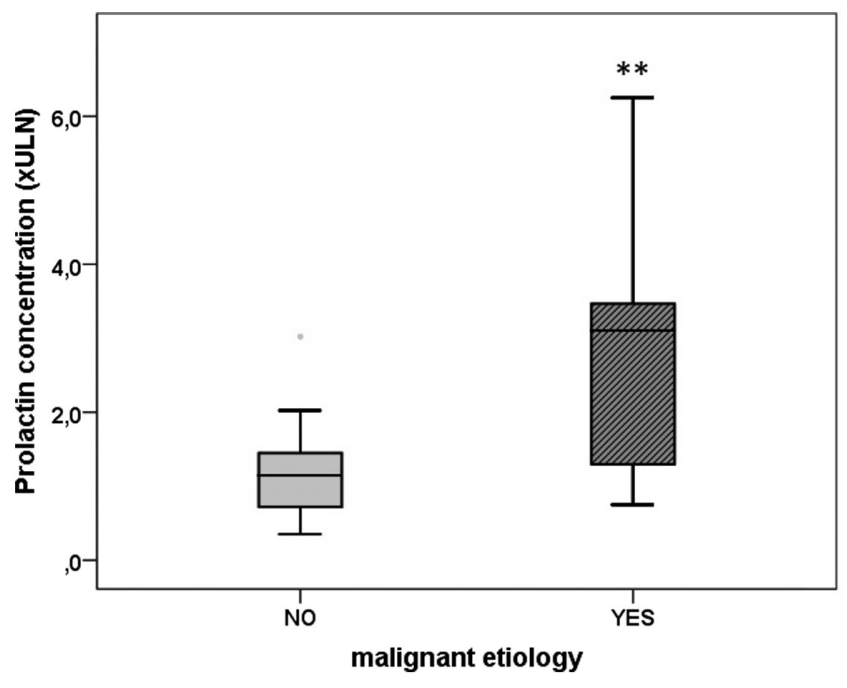

Figure 2

Serum prolactin concentration expressed as a multiple of the upper limit of normal ( $\times U L N)$ in patients with a benign (open bar) or malignant etiology of pituitary stalk thickening (dark grey bar). Boxplots represent the medians, interquartile ranges and (P5-P95) intervals. $* * P<0.01$ between the two groups.

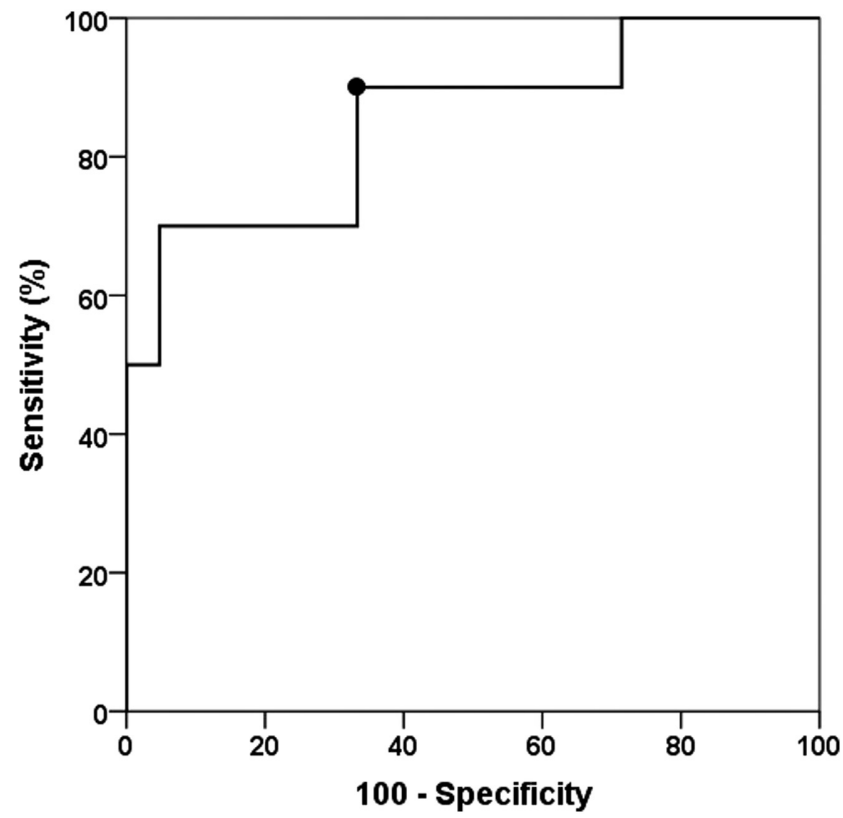

Figure 3

ROC curve analysis of prolactin concentration for the differentiation between neoplastic and benign causes of pituitary stalk thickening. The best cut off value for serum prolactin (designed by the filled circle) was $1.27 \times U L N$ with a sensitivity of $90 \%$ and a specificity of $67 \%$ (AUC $=0.852$; $P=0.002)$. 
Table 5 Treatment characteristics and MRI evolution according to the malignant nature of underlying disease.

\begin{tabular}{l}
\hline Treatment of underlying disease (\%) \\
Beneficial effects on stalk thickening (\%) \\
Nb of patients with PS normalization (\%) \\
Time to stalk normalization (months) \\
\hline
\end{tabular}

\begin{tabular}{c}
\hline Benign disease $(n=27)$ \\
\hline $14 / 27(52 \%)$ \\
$12 / 12$ \\
$14 / 24(58 \%)$ \\
$31(5-206)$ \\
\hline
\end{tabular}

\begin{tabular}{c}
\hline Malignant disease $(n=11)$ \\
\hline $10 / 11(91 \%)$ \\
$8 / 9$ \\
$9 / 9(100 \%)$ \\
$7(3-18)$
\end{tabular}

\begin{tabular}{c}
\hline P value \\
\hline $\mathbf{0 . 0 1 4}$ \\
0.262 \\
$\mathbf{0 . 0 4 7}$ \\
$\mathbf{0 . 0 1 5}$ \\
\hline
\end{tabular}

$\mathrm{Nb}$, number.

spontaneous normalization of pituitary stalk thickness in one and a further enlargement in the other. At the end of the observation period, regardless of treatment or normalization of the pituitary stalk, CDI persisted in all patients except in one suffering from leukemia and treated by bone marrow cell allotransplantation. Likewise, initial anterior pituitary deficits persisted at the last follow-up visit, with gonadotropic, somatotropic, thyrotropic and corticotropic deficits being present at that time in $20 / 33$ (61\%); 21/28 (75\%), 21/34 (62\%) and 12/32 (38\%) patients, respectively.

\section{Discussion}

In this retrospective study, we describe the etiological spectrum of diseases responsible for the association of CDI and PST in patients over the age of 15 years. We also highlight the common diagnostic strategy used in our three centers when facing this condition and compare the main characteristics and outcome of neoplastic and non-neoplastic diseases involving the pituitary stalk in adult patients.

In our study, the risk of having a malignant disease when CDI and PST were associated was 29\% (11/28 patients). Comparison with other similar reports is difficult since many studies also included pediatric cases, considered any pituitary stalk lesion whether associated or not with CDI, and/or included cases without a welldefined diagnosis $(1,3,8,9,14)$. As reported by Catford et al. (20), the nature of PS lesions indeed differs between small case series or compiled cases reports and larger monocentric series. In their review of the literature in 2016, the authors identified 209 published cases of PST in both adults and children and estimated the relative proportion of congenital, inflammatory or infectious, and neoplastic causes to be 3, 68 and $29 \%$, respectively. Conversely, in three of the largest studies $(1,3,14)$, neoplastic diseases were more frequent, often accounting for the majority of cases in which a pathological diagnosis was established, with a reported frequency rate between 37 and 59\%. One should however keep in mind that the overall biopsy rate in these series was rather low (between 24 and 45\%), and that malignant lesions were likely more often biopsied. More recently, the largest monocentric study to date was published by Zou et al. (13), reporting on 321 Chinese patients with PST. Again, a high proportion of neoplasms (75\%) was reported among the patients who had histologically proven disease, with similar low biopsy rates of $43 \%$ overall and of $29 \%$ for pituitary stalk biopsies. Moreover, the high relative proportion of neoplastic etiologies was also explained by a large proportion of patients with undetermined etiology ('idiopathic PST'), which probably represents mostly autoimmune neuroinfundibulitis) and by the misclassification of Langerhans cell type (L) histiocytosis as myeloid neoplasia, thus accounting for more than half of the malignant neoplastic PST. In the present study, the overall biopsy rate (whatever the organ biopsied) was somewhat higher than in most other studies (58\%) and the malignancy rate was lower (29\%), likely because we considered L-histiocytosis as a benign entity and we did not include the patients with a previously known metastatic malignancy. Both conditions are frequently described as etiologies of malignant PST in adults patients $(3,13)$.

The only study that addressed the neoplastic risk in patients with PST and CDI by performing systematic surgical pituitary biopsies showed comparable results (14). In this previous report from Jian and colleagues, $84 \%$ of their 37 patients were older than 15 , a pathological diagnosis was established for $17 / 37$ patients (46\%) and the three most frequent etiologies were idiopathic PST for 20 patients (54\%), germinoma for 9 patients (24\%) and histiocytosis for 7 patients (19\%).

When facing such association of CDI and PST, the main goals of the medical workup are to exclude a neoplastic underlying pathology, to determine the presence of associated pituitary deficits and to propose the appropriate medical therapy. All possible clues to narrow the etiological spectrum are therefore welcome. In this regard, we observed that male gender and a very young age were statistically associated with a risk of neoplasia, essentially related to the higher incidence of germ cell 
tumor in males and their occurrence mostly before the end of the third decade of life (21). In contrast, metastatic spread and inflammatory diseases are more frequent in adults (13). Whether the presence of concomitant CDI increases or not the risk of neoplasm is controversial. Some authors have suggested that CDI is a negative predictor of neoplastic PST, being thus a favorable factor (11) while others have shown the opposite $(2,22)$. Obviously, our study including only patients with CDI was unable to solve this dilemma.

A thorough clinical examination remains also of utmost importance. Cutaneous lesions such as nodules or generalized crusted papules should evoke histiocytosis and were indeed present in most of our patients with this disease, but never observed in the other cases. Neurologic signs were only present in patients with a germ cell tumor. Other relevant clinical features include lymphadenopathy, hepatosplenomegaly or other features of a systemic disease. Performing an FDG-PET-CT scan will usually help the clinician to localize extra-cerebral localizations in such instances and guide the diagnostic biopsy procedure (13).

Brain imaging is obviously indicated in each patient with CDI, but we could not identify a pathognomonic shape of the stalk or other features that could help the clinician to identify the underlying disease with a sufficient degree of confidence. In the study of Turcu et al., the strongest association with a specific pattern of stalk thickening on MRI was described for congenital lesions which were usually round in appearance. Most cases of neurosarcoidosis had a uniformly thickened pituitary stalk and most metastases of solid cancers appeared round or V-shaped. There was however no correlation between a specific stalk pattern and either germinoma, Langerhans cell histiocytosis, Erdhem Chester disease, Wegener hypophysitis or lymphocytic hypophysitis (3). As already observed by Sbardella et al. (2) and by Lee et al. (11), the pituitary stalk thickness was larger in our patients with a neoplastic etiology of PST, in particular those with a germinoma, but a large overlap was observed with benign lesions. It should also be pointed out that, as opposed to our study, previous reports included congenital and cystic tumors such as craniopharyngiomas or Rathke's cleft cysts in their study population, which may significantly influence the findings. The only decisive clue provided by imaging was the presence of a concomitant pineal lesion in three patients with a germ cell tumor. Of note, CSF analysis was useful for the diagnosis of germinomas as $6 / 8$ patients had positive hCG or typical neoplastic cells in their CSF, and diagnosis only relied on this analysis in one patient. Intracranial germ cell tumors can secrete tumor markers such as hCG and AFP into serum and/or CSF. However, discordant serum and CSF tumor marker results have been observed and CSF analysis is needed for an accurate diagnosis since protein levels are often much higher in CSF than in serum. Moreover, we should keep in mind that, as shown in our study, pure germinomas typically present with normal hCG and AFP levels in both serum and CSF. Therefore, the negativity of these biomarkers does not rule out the diagnosis which requires histological confirmation if suspected (23).

Complete pituitary endocrine evaluation is also mandatory in each patient with PST in order to assess whether an endocrine deficit is present that needs to be treated. Data concerning the relation between neoplastic etiology and endocrine deficits are conflicting.

In our study, at least one anterior pituitary deficit was observed in nearly $60 \%$ of patients but the number of endocrine deficits was clearly related to the risk of neoplasia. Indeed, one or more deficits were observed in $100 \%$ of patients with neoplastic etiology but only in $41 \%$ of the remaining patients. When two or more anterior pituitary deficits were observed, the risk of malignancy was superior to $90 \%$ compared to only $20 \%$ in benign diseases. Secondary hypogonadism was the most common endocrine deficiency in both benign and neoplastic lesions as also observed in previous reports (2, $3,13,14)$. Gonadotropic deficiency could however be the result of either the deleterious effect of hypothalamic, stalk or pituitary lesions, or the functional consequence of associated hyperprolactinemia.

There are limited data about hyperprolactinemia in pituitary stalk lesions. Hyper-prolactinemia was detected in respectively $19 \%$ and $30 \%$ of the patients in the studies of Doknic (10) and Turcu (3). According to Lee and colleagues (11), the presence of hyperprolactinemia was not a useful parameter for differentiating neoplastic from non-neoplastic lesions. However, they gave no information about absolute prolactin levels in their report. Interestingly, in our study, a high median PRL serum level was individually the best predictor of a neoplastic origin with an optimal cutoff value of $1.27 \times$ the upper limit of normal, predicting malignancy with a sensitivity of $90 \%$ and a specificity of $67 \%$. PRL level was also though non-statistically - correlated with the thickness of pituitary stalk disease, meaning that the magnitude of hyperprolactinemia could reflect the severity of the stalk infiltration. Of note, our study is the first to bring out high prolactin levels as a risk factor for a neoplastic etiology. This is probably due to the fact that prolactin 
measurement is often not considered as mandatory as it will not imply substitution therapy.

Despite a complete work-up, etiology of PST may remain unknown with the risk of ignoring a neoplastic lesion. Therefore, when the etiology is unclear, the possibility of malignancy cannot be formally excluded, and if no biopsy is feasible at another extracerebral site, a pituitary stalk biopsy needs to be considered. In fact, two different approaches have been suggested in such instances $(3,8,13,14,20)$ : either a transsphenoidal biopsy of the stalk to allow early histopathological diagnosis, or very close (3-6 months) imaging follow-up and later biopsy if disease is progressing. In our study, stalk biopsy was finally performed in only a minority $(3 / 27)$ of patients with a benign disease and in $4 / 11$ with a neoplastic disease. This illustrates well the fact that stalk biopsy should rarely be proposed for neuroinfundibulitis and always proposed in case of suspicion of a neoplastic etiology that cannot be confirmed by other procedures.
Taken all these facts together, our suggested approach for the diagnosis and management of PST and CDI is outlined in Fig. 4.

Data recorded on the follow-up of our patients (median 55 months) also allowed us to reinforce the primary diagnosis of benign etiology in case of stable or spontaneously improving disease. Progressive increase in the size of pituitary stalk lesion is well described in the natural course of germ cell tumor or LCH $(14,24)$. On the other hand, spontaneous regression of PST is a common feature of lymphocytic neuroinfundibulitis and may also occur, although not commonly - in isolated pituitary stalk localization of LCH (6). Finally, at the end of follow-up, pituitary stalk abnormalities persisted in about one third of the patients, mainly in those with a benign etiology. A complete normalization of pituitary stalk appearance was indeed observed in all malignant PST and this can be explained by early aggressive treatments including radiotherapy for all, combined with chemotherapy,

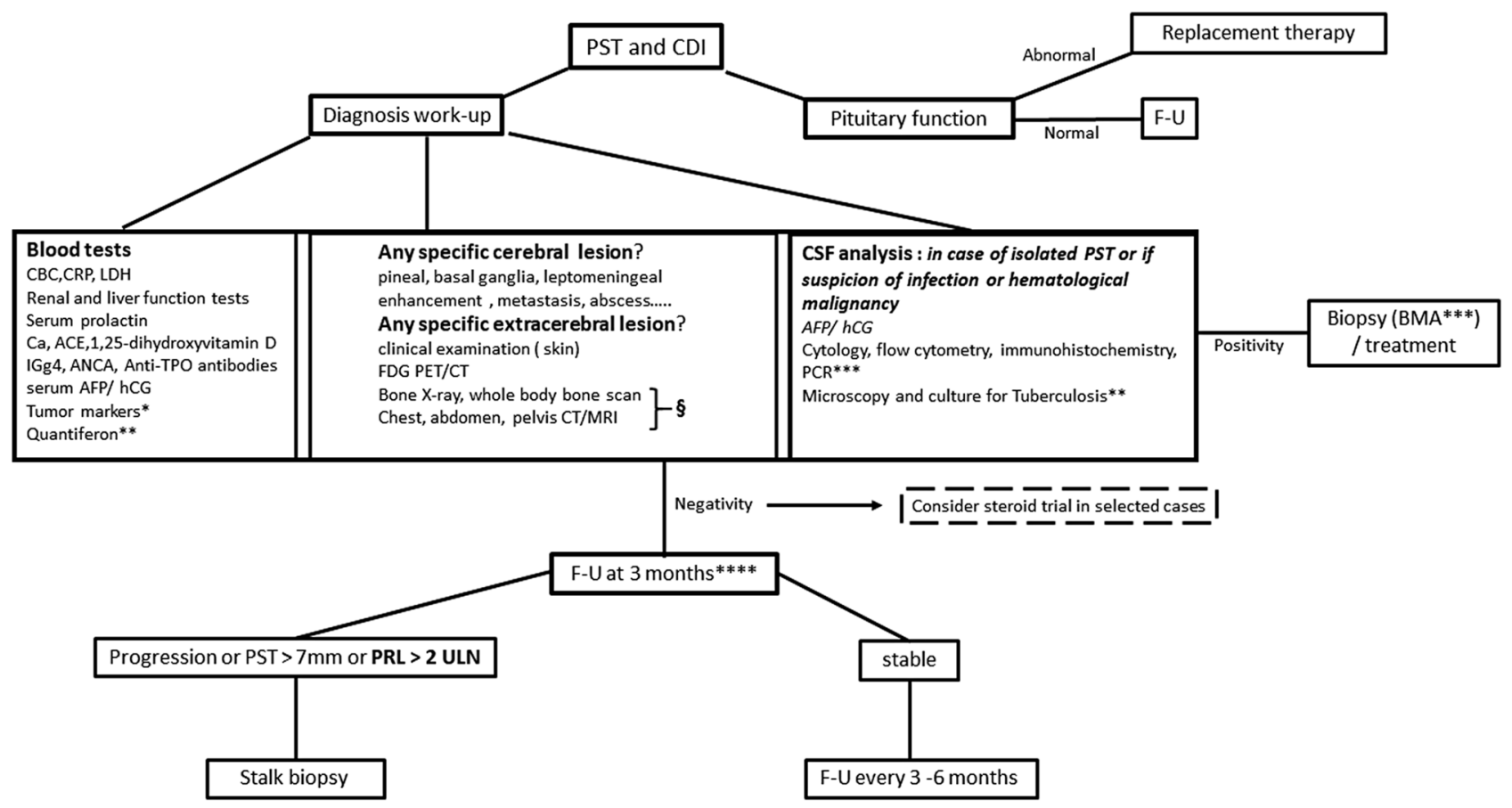

\section{Figure 4}

Suggested approach for the diagnosis and management of pituitary stalk thickening (PST) and central diabetes insipidus (CDI). ACE: angiotensin-converting enzyme, AFP: $\alpha$-fetoprotein, ANCA: antineutrophil cytoplasmic antibody, Anti-TPO: anti peroxidase, BMA: bone marrow aspirate, CBC: complete blood cell count, CDI: central diabetes insipidus, CRP: C-reactive protein, CSF: cerebrospinal fluid, F-U: follow-up, hCG: human chorionic gonadotropin, LDH: lactate dehydrogenase, PCR: polymerase chain reaction, PRL: prolactin, PST: pituitary stalk thickening, ULN: upper limit of normal. *For selected patients in whom metastatic malignancy is suspected; **for selected patients in whom tuberculosis is suspected (most often immunocompromised patients or endemic areas); ***for patients with suspected lymphoma (BMA for confirmation and staging of the disease); $* \star \star * c e r e b r a l / s e l l a r$ MRI and pituitary function; ${ }^{\S}$ depending on PET-CT availability and results. 
hormone therapy and bone marrow allotransplantation in one patient with leukaemia.

Despite normalization of PST in the majority of patients, CDI persisted in all but one of them, thus suggesting the irreversible destruction of vasopressin secreting neurons. Similar data have been reported previously. For example, Jian et al. reported the persistence of CDI throughout the clinical course of all their patients, with a median follow-up of more than 5 years, including those with complete regression of pituitary stalk lesions. Similar findings were observed for anterior pituitary deficits when present at diagnosis.

There are several limitations to be acknowledged regarding our study, mainly the lack of tissue-confirmed diagnosis, a pre-determined intrinsic selection bias and a small number of patients. Further studies with systematic measurement of prolactin concentrations are also needed to confirm our findings.

In conclusion, our study confirms the relatively high risk of malignancy in adult patients presenting with pituitary stalk thickening and central diabetes insipidus. The differential diagnosis is however wide and must include germ cell tumors, solid organ metastases, benign lymphocytic neuroinfundibulitis or Langerhans cell type histiocytosis among the most prevalent causes. Early diagnosis of germinoma remains particularly challenging. Young age, male gender, a very large thickening of the stalk (without evidence of a cystic tumor), a higher number of anterior pituitary deficits as well as a serum level of prolactin above 1.3 times the upper limit of normal are all non-invasive parameters increasing the likelihood of a neoplastic origin.

\section{Declaration of interest}

The authors declare that there is no conflict of interest that could be perceived as prejudicing the impartiality of this study.

\section{Funding}

This research did not receive any specific grant from any funding agency in the public, commercial or not-for-profit sector.

\section{Acknowledgements}

The authors thank their colleagues, neuroradiologist and clinicians for their valuable collaboration on this manuscript.

\section{References}

1 Hamilton BE, Salzman KL \& Osborn AG. Anatomic and pathologic spectrum of pituitary infundibulum lesions. American Journal of
Roentgenology 2007188 W223-W232. (https://doi.org/10.2214/ AJR.05.2027)

2 Sbardella E, Joseph RN, Jafar-Mohammadi B, Isidori AM, Cudlip S \& Grossman AB. Pituitary stalk thickening: the role of an innovative MRI imaging analysis which may assist in determining clinical management. European Journal of Endocrinology 2016175 255-263. (https://doi.org/10.1530/EJE-16-0455)

3 Turcu AF, Erickson BJ, Lin E, Guadalix S, Schwartz K, Scheithauer BW, Atkinson JL \& Young Jr WF. Pituitary stalk lesions: the Mayo Clinic experience. Journal of Clinical Endocrinology and Metabolism 201398 1812-1818. (https://doi.org/10.1210/jc.2012-4171)

4 Liu W, Wang L, Liu M \& Li G. Pituitary morphology and function in 43 children with central diabetes insipidus. International Journal of Endocrinology 20162016 6365830. (https://doi. org/10.1155/2016/6365830)

5 De Buyst J , Massa G , Christophe C, Tenoutasse S \& Heinrichs C. Clinical, hormonal and imaging findings in 27 children with central diabetes insipidus. European Journal of Pediatrics 2007166 43-49. (https://doi.org/10.1007/s00431-006-0206-0)

6 Leger J, Velasquez A, Garel C, Hassan M \& Czernichow P. Thickened pituitary stalk on magnetic resonance imaging in children with central diabetes insipidus. Journal of Clinical Endocrinology and Metabolism 199984 1954-1960. (https://doi.org/10.1210/ jcem.84.6.5745)

7 Maghnie M, Cosi G, Genovese E, Manca ML, Cohen A, Zecca S, Tinelli C,Galluci M, Bernasconi S,Boscherini B et al. Central diabetes insipidus in children and young adults. New England Journal of Medicine 2000343 998-1007. (https://doi.org/10.1056/ NEJM200010053431403)

8 Jinguji S, Nishiyama K, Yoshimura J, Yoneoka Y, Harada A, Sano M \& Fujii Y. Endoscopic biopsies of lesions associated with a thickened pituitary stalk. Acta Neurochirurgica 2013155 119-124; discussion 124. (https://doi.org/10.1007/s00701-012-1543-6)

9 Trabelsi L, Mnif M, Rekik N, Kaffel N, Charfi N, Mnif J, Kchaow MS \& Abid M. MRI pituitary stalk abnormalities: etiology aspects in 11 patients. Annales d'Endocrinologie 200667 604-612. (https://doi. org/10.1016/s0003-4266(06)73014-1)

10 Doknic M, Milijic D, Pekic S, Stojanovic M, Savic D, Manojlovic E, Milenkovic T, Zdravkovic V,Jesic M, Damjanovic D et al. Single center study of patients with pituitary stalk lesions. Pituitary 201821 605-614. (https://doi.org/10.1007/s11102-018-0914-2)

11 Lee JY, Park JE, Shim WH, Jung SC, Choi CG, Kim SJ, Kim JH \& Kim HS. Joint approach based on clinical and imaging features to distinguish non-neoplastic from neoplastic pituitary stalk lesions. PLOS ONE 201712 e0187989. (https://doi.org/10.1371/journal. pone.0187989)

12 Rupp D \& Molitch M. Pituitary stalk lesions. Current Opinion in Endocrinology, Diabetes, and Obesity 200815 339-345. (https://doi. org/10.1097/MED.0b013e3283050844)

13 Zhou X, Zhu H, Yao Y, Lian X, Feng F, Wang L, Liu S, Deng K, You H, Yang $\mathrm{H}$ et al. Etiological spectrum and pattern of change in pituitary stalk thickening: experience in 321 patients. Journal of Clinical Endocrinology and Metabolism 2019104 3419-3427. (https://doi. org/10.1210/jc.2018-02297)

14 Jian F, Bian L, Sun S, Yang J, Chen X, Chen Y, Ma Q, Miao F, Wang W, Ning $\mathrm{G}$ et al. Surgical biopsies in patients with central diabetes insipidus and thickened pituitary stalks. Endocrine 201447 325-335. (https://doi.org/10.1007/s12020-014-0184-3)

15 Simmons GE, Suchnicki JE, Rak KM \& Damiano TR. MR imaging of the pituitary stalk: size, shape and enhancement pattern. American Journal of Roentgenology 1992159 375-377. (https://doi.org/10.2214/ ajr.159.2.1632360)

16 Raveendranath V, Nagarajan K, Umamageswari A, Srinidhi S $\&$ Kavitha T. Three-dimensional magnetic resonance-based morphometry of pituitary stalk. Radiologia Medica 2019124 206-210. (https://doi.org/10.1007/s11547-018-0956-6) 
17 Satogami N, Miki Y, Koyama T, Kataoka M \& Togashi K. Normal pituitary stalk: high-resolution MR imaging at 3T. American Journal of Neuroradiology 201031 355-359. (https://doi.org/10.3174/ajnr. A1836)

18 Tien RD, Newton TH, McDermott MW, Dillon WP \& Kucharczyk J. Thickened pituitary stalk on MR images in patients with diabetes insipidus and Langerhans cell histiocytosis. American Journal of Neuroradiology 199011 703-708.

19 Emile JF, Abla O, Fraitag S, Horne A, Haroche J, Donadieu J, RequenaCaballero L, Jordan MB, Abdel-Wahab O, Allen CE et al. Histiocyte Society. Revised classification of histiocytosis and neoplasms of the macrophage-dendritic cell lineages. Blood 2016127 2672-2681. (https://doi.org/10.1182/blood-2016-01-690636)

20 Catford S, Wang YY \& Wong R. Pituitary stalk lesions: systematic review and clinical guidance. Clinical Endocrinology 201685 507-521. (https://doi.org/10.1111/cen.13058)
21 Fetcko K \& Dey M. Primary central nervous system germ cell tumors: a review and update. Medical Research Archives 20186 1719. (https:// doi.org/10.18103/mra.v6i3.1719)

22 Robison NJ, Prabhu SP, Sun P, Chi SN, Kieran MW, Manley PE, Cohen LE, Goumnerova L, Smith ER, Scott RM et al. Predictors of neoplastic disease in children with isolated pituitary stalk thickening. Pediatric Blood and Cancer 201360 1630-1635. (https:// doi.org/10.1002/pbc.24577)

23 Osorio DS \& Allen JC. Management of CNS germinoma. CNS Oncology 20154 273-279. (https://doi.org/10.2217/cns.15.13)

24 Di Iorgi N, Allegri AE, Napoli F, Calcagno A, Calandra E, Fratangeli N, Vannati M, Rossi A, Bagnasco F, Haupt R et al. Central diabetes insipidus in children and young adults: etiological diagnosis and long-term outcome of idiopathic cases. Journal of Clinical Endocrinology and Metabolism 201499 1264-1272. (https://doi. org/10.1210/jc.2013-3724)

Received 25 January 2020

Revised version received 7 April 2020

Accepted 29 April 2020 